\title{
Prognostic Factors For Outcome in Patients with Refractory and Relapsed Acute Lymphocytic Leukemia Treated with Inotuzumab Ozogamicin, a CD22 Monoclonal Antibody
}

\author{
Elias Jabbour ${ }^{1}$, Susan O'Brien ${ }^{1}$, Xuelin Huang ${ }^{2}$, Deborah Thomas ${ }^{1}$, Michael Rytting ${ }^{1}$, Koji \\ Sasaki $^{1}$, Jorge Cortes ${ }^{1}$, Guillermo Garcia-Manero ${ }^{1}$, Tapan Kadia ${ }^{1}$, Farhad Ravandi ${ }^{1}$, Sherry \\ Pierce $^{1}$, and Hagop Kantarjian ${ }^{1}$ \\ ${ }^{1}$ Department of Leukemia, U.T. M.D. Anderson Cancer Center, Houston, TX \\ ${ }^{2}$ Department of Biostatistics, U.T. M.D. Anderson Cancer Center, Houston, TX
}

\begin{abstract}
Background-Inotuzumab ozogamicin was found to be highly active in patients with refractoryrelapsed acute lymphocytic leukemia (ALL), with an overall response rate of 58\% and a median survival of 6.3 months. Identifying factors associated with different outcomes on inotuzumab therapy may help select patients for this treatment and advice of prognosis.
\end{abstract}

Methods-A total of 89 patients treated with inotuzumab on previous studies were analyzed. Inotuzumab was given at $1.3-1.8 \mathrm{mg} / \mathrm{m}^{2}$ intravenously (IV) x 1 every 3-4 weeks or weekly (0.8 $\mathrm{mg} / \mathrm{m}^{2}$ Day $1,0.5 \mathrm{mg} / \mathrm{m}^{2}$ Days 8 and 15 ) every 3-4 weeks. Pretreatment factors associated with achieving marrow complete response (CR) and with survival were analyzed using standard statistical methods.

Results-The median survival of patients with at least marrow CR was 9.2 months versus 3.4 months for those without marrow $\mathrm{CR}(\mathrm{p}<0.001)$. By multivariate analysis, a high peripheral blood absolute blast count and low platelet count were independently associated with a lower likelihood of achieving at least marrow CR. Baseline characteristics independently associated with worse survival included adverse cytogenetics [complex karyotype, translocation $(4 ; 11)$, translocation (9;22), abnormal chromosome 17], disease beyond first salvage, and high peripheral blood absolute count. Patients with $0,1-2$ or 3 adverse factors had a median survival of 39+, 7.5, and 2.4 months, respectively.

Conclusions-Our current analyses identified a subset of adult patients with ALL in whom outcome of therapy with inotuzumab ozogamicin can be differentially predicted.

\section{Keywords}

acute lymphocytic leukemia; relapse/refractory; inotuzumab ozogamicin; outcome

Address Correspondence: Elias Jabbour, MD, Department of Leukemia, The University of Texas, M.D. Anderson Cancer Center, 1515 Holcombe Blvd. Box 428. Houston, TX 77030, Phone: 713-792-4764; Fax: 713-794-4297; ejabbour@ mdanderson.org. 


\section{INTRODUCTION}

Targeted therapies provide novel approaches in the treatment of acute lymphocytic leukemia (ALL), either with tyrosine-kinase inhibitors to target fusion proteins, or immunotherapies with antibodies directed toward cell surface antigens-eg, CD19, CD20, or CD22. ${ }^{1}$ CD22 is among the most frequently expressed surface antigens in B-precursor ALL and is expressed on immature and mature B cells, but not on hematopoietic stem cells. It is therefore an ideal target for immunotherapy. ${ }^{2} \mathrm{CD} 22$ is also a suitable target for immunoconjugated toxins that can restrict intracellularly their cytotoxic effect against target cells. ${ }^{2}$ Inotuzumab ozogamicin is a CD22 monoclonal antibody bound to calicheamicin, a natural product of Micromonospora echinospora, which is significantly more toxic than cytotoxic chemotherapy. ${ }^{3}$ Inotuzumab binds CD22 with subnanomolar affinity and is rapidly internalized, delivering the conjugated calicheamicin intracellularly. Calicheamicin binds to the minor DNA groove, causing double-strand DNA breaks and resulting in cell apoptosis.

Inotuzumab ozogamicin was evaluated in 89 patients with refractory-relapsed ALL and was found to be highly active, with an overall response rate of 58\%. ${ }^{4-5}$ However responses were not durable, and median survival was 6.3 months. ${ }^{4-5}$ The purpose of the analysis was to identify baseline patient and disease factors that could potentially predict for differences in outcomes.

\section{MATERIALS AND METHODS}

\section{Study Group}

Ninety patients with a confirmed diagnosis of refractory or relapsed pre-B ALL were eligible. One patient was treated sequentially with the 2 schedules. He was initially treated with the single dose schedule, responded and received allogeneic stem cell transplantation. Unfortunately, he relapsed post transplantation and received the weekly regimen. For the purpose of the current study, he was counted one time as treated in the single dose schedule. Eligibility criteria were identical for the single-dose $(n=49)$ and weekly $(n=40)$ inotuzumab schedules and were previously reported. ${ }^{4-5}$

This was a single-institution study conducted at The University of Texas MD Anderson Cancer Center. The study protocols were approved by the Institutional Review Board, in compliance with institutional guidelines. Patients signed informed consent in compliance with the Declaration of Helsinki.

\section{Therapy}

Patients received inotuzumab from 1.3 to $1.8 \mathrm{mg} / \mathrm{m}^{2}$ as a one-hour intravenous infusion once every 3 to 4 weeks. Weekly inotuzumab was given at a dose of $0.8 \mathrm{mg} / \mathrm{m}^{2}$ on day 1 and 0.5 $\mathrm{mg} / \mathrm{m}^{2}$ on days 8 and 15 , for a total dose of $1.8 \mathrm{mg} / \mathrm{m}^{2}$ per course. Courses were repeated every 3 to 4 weeks. Inotuzumab was given as a short infusion over 1 hour. Courses were given every 3-4 weeks, depending on the recovery of the counts. Persistent thrombocytopenia was not a condition to delay therapy. Patients who achieved a complete response (CR) or a marrow $\mathrm{CR}$ after a maximum of 4 courses were allowed to receive 2 additional courses of therapy, for a maximum of 6 cycles. Additional treatments were based 
on response and liver toxicities in the previous 4 cycles. Patients with grade 3 or worse toxicity and a favorable response to therapy could receive inotuzumab in subsequent cycles at a 25\% dose reduction. Patients who developed central nervous system (CNS) leukemia on inotuzumab and who had a positive response were allowed to continue on therapy and receive CNS-directed intrathecal chemotherapy after assessment of the patient benefit:risk ratio.

Suitability for allogeneic stem cell transplantation (ASCT) was assessed in all patients. Whenever possible, eligible patients were considered for ASCT after achieving at least marrow CR.

\section{Statistical Considerations}

Response criteria were standard. A CR was defined as the disappearance of all disease with bone marrow blasts $5 \%$, neutrophils $\geq 1.0 \times 10^{9} / \mathrm{L}$, and a platelet count $>100 \times 10^{9} / \mathrm{L}$. A marrow $\mathrm{CR}$ with incomplete recovery of platelets $(\mathrm{CRp})$ was defined as a $\mathrm{CR}$ but without platelet recovery to $\geq 100 \times 10^{9} / \mathrm{L}$. A bone marrow CR with incomplete recovery (CRi) was defined as CR but without recovery of platelets to $\geq 100 \times 10^{9} / \mathrm{L}$ or recovery of neutrophil counts to $\geq 1.0 \times 10^{9} / \mathrm{L}$.

Differences among variables were evaluated by the Chi-square test and Mann-Whitney U test for categorical and continuous variables, respectively. Overall survival was defined as the time interval between the start of therapy date and last follow-up or date of death.

Patients were not censored when they were taken off study. Patients who were alive were censored at the last follow-up date. The probabilities of survival were estimated using the method of Kaplan and Meier. Univariate and multivariate analyses were performed to identify potential factors associated with the achievement of marrow CR and survival. Factors retaining significance in the multivariate models were interpreted as being independently predictive of the achievement of response or survival. Multivariate analysis of response used logistic regression model and survival used the Cox proportional hazard regression model. ${ }^{6-8} \mathrm{~A}$ p-value of $<0.05$ (two-tailed) was considered statistically significant.

\section{RESULTS}

\section{Baseline patient characteristics}

Baseline characteristics of the 89 patients are summarized in Table 1. Forty-nine patients received single-dose inotuzumab and were accrued between June 2010 and March 2011; 40 received weekly inotuzumab and were accrued between March 2011 and September 2012. Their median age was 39 years (range, $4-84$ years). All patients expressed high levels of CD22 positivity in $250 \%$ leukemic cells.

\section{Response to inotuzumab ozogamicin}

Overall, 17 patients (19\%) achieved CR, 29 patients (33\%) had CRp, and 6 patients (7\%) had CRi. Thirty-two patients (36\%) had resistant disease, and 5 patients (6\%) died within 4 weeks of starting therapy. Thus, responses were observed in 52 of the 89 patients who received treatment, for an overall response rate of 58\%. Response rates with weekly and 
single-dose inotuzumab were similar (Table 2). The median number of cycles was 2 (range, 1-5 cycles) with weekly and 2 (range, 1-6 cycles) with single-dose inotuzumab. The median number of cycles to response was 1 (median, 1-4 cycles).

Among the 52 patients who achieved a response, $36(69 \%)$ had chromosomal abnormalities at the start of therapy and had sufficient metaphases for analysis at morphologic CR. Among these, a cytogenetic CR was observed in 28 patients (78\%). The rate of cytogenetic CR by morphologic responses was as follows: 8 cytogenetic CR/10 morphologic CR (80\%); 16 cytogenetic CR/22 morphologic CRp (73\%); and 4 cytogenetic CR/4 morphologic CRi $(100 \%)$.

Most patients who achieved CR obtained it early (there were 13 CRs after 1 course and 4 CRs after 2 or more courses). Among the patients who achieved a CRp, 16 achieved it after 1 course, and 13 achieved it after 2 or more courses. Among the patients who achieved a CRi, 1 achieved it after 1 course, and 5 achieved it after 2 or more courses. Ten patients received additional rituximab. Of them, four patients did not initially respond to inotuzumab. Only one of them did improve his response from no response to CRi.

\section{Factors associated with the achievement of a marrow response}

Since the achievement of a response was reported to be associated with improved outcome, ${ }^{9-10}$ we analyzed the correlation between patient characteristics and the achievement of at least marrow CR (Table 3).

By univariate analysis, the presence of complex karyotype, translocation $(4 ; 11)$, translocation $(9 ; 22)$, and abnormal chromosome 17, in addition to Salvage 2 and beyond, high white blood cell count ( $\geq 4.0 \times 10^{9} / \mathrm{L}$ ), high absolute peripheral blood blast count $\left[(\mathrm{ABC}) ; \geq 1.0 \times 10^{9} / \mathrm{L}\right]$, and low platelet count $\left(<100 \times 10^{9} / \mathrm{L}\right)$ were associated with a lower likelihood of achievement of a marrow CR. By multivariate analysis, a high ABC $[\mathrm{OR}=6.9$ (95\% CI=5.7-7.9); $\mathrm{p}<0.001]$ and low platelet count [OR=11.5 (95\% CI=9.3-13.7); $\mathrm{p}=0.03$ ] were independently associated with a lower likelihood of achieving a marrow CR. In multivariate analysis, there was no difference in the odds of achieving a response whether the disease was in Salvage 1 or Salvage 2 and beyond.

\section{Outcome and prognostic factors for survival}

With a median follow-up of 23 months, (range, 5 to 44 months), the median overall survival of patients $(n=89)$ who received inotuzumab was 6.3 months; the median survival was 5.0 months with the single-dose schedule and 9.5 months with the weekly schedule. The median remission duration for responding patients $(\mathrm{n}=52)$ was 11.7 months (range, $1-43 ; 1$-year rate, $48 \%$ ) (Figure 1).

By univariate analysis (Table 3$)$, patients with complex karyotype, translocation $(4 ; 11)$, translocation $(9 ; 22)$, and abnormal chromosome 17 had worse survival (median 5.0 months compared to 44 months + for the others; $\mathrm{p}<0.001)$; patients who received treatment in Salvage 1 had a median survival of 10.2 months compared with 4.8 months for those receiving treatment in Salvage 2 and beyond ( $\mathrm{p}<0.001)$; patients with high $\mathrm{ABC}(\geq 1.0 \mathrm{x}$ $\left.10^{9} / \mathrm{L}\right)$ and low platelets count $\left(<100 \times 10^{9} / \mathrm{L}\right)$ had a worse survival as well. 
By multivariate analysis, baseline characteristics independently associated with worse survival included adverse cytogenetics [complex karyotype, translocation $(4 ; 11)$, translocation $(9 ; 22)$, and abnormal chromosome 17] [HR=2.9 (95\% CI=1.27-6.46); $\mathrm{p}=0.01]$, disease beyond Salvage 1 [HR=2.3 (95\% CI=1.24-4.09); $\mathrm{p}=0.007]$, and high $\mathrm{ABC} \geq 1.0 \mathrm{x}$ $10^{9} / \mathrm{L}[\mathrm{HR}=1.8(95 \% \mathrm{CI}=1.06-2.93) ; \mathrm{p}=0.02]$. While, the weekly schedule was shown to be better than the single-dose schedule in the univariate analysis, $(\mathrm{p}=0.03)$, it did not retain its advantage in the multivariate model after adjusting for all other variables $(\mathrm{HR}=0.63$; $\mathrm{p}=0.11$ ). Since the relative impact of each of these 3 factors on survival was similar, we assigned an arbitrary value of 1 to each of them. Patients with $0(n=6), 1(n=25), 2(n=35)$ or $3(\mathrm{n}=22)$ adverse factors had a median survival of 39+, 7.6, 7.4, and 2.4 months, respectively (Figure 2).

In a subsequent time, we validated the prognostic scoring system identified into a historical cohort of 253 patients with relapsed/refractory ALL treated at our institution. The model identified was found to be predictive for survival in this control group. The 18-months survival rates were $25 \%, 7 \%, 6 \%$, and $0 \%$, for patients with $0(n=60), 1(n=104), 2(n=68)$, or $3(\mathrm{n}=21)$ adverse factors, respectively $(\mathrm{p}<0.001)$.

\section{Achievement of marrow CR and prognosis}

To assess the benefit of achieving a marrow $\mathrm{CR}$, we repeated the multivariate survival analysis using a 6-week landmark that excluded 5 patients who died within 6 weeks. The 6week time period was chosen to reduce the bias that could result from patients who experienced early death and as such were not evaluable for response. Furthermore, the response reported is the best response observed. Five of the 83 patients assessed achieved their best response (CR) after 6 weeks: two were in CRi and three required three courses to achieve response. The median survival was 9.2 months and 3.4 months for patients with and without marrow CR $(\mathrm{p}<0.001)$. The multivariate analysis included 83 patients and selected the achievement of response as independently associated with survival improvement $[\mathrm{HR}=0.5$ (95\% CI=0.28-0.89); $\mathrm{p}=0.02]$. Disease with complex karyotype, translocation $(4 ; 11)$, translocation $(9 ; 22)$, and abnormal chromosome 17 [HR=2.9 (95\% CI=1.29-6.62); $\mathrm{p}=0.009$ ], and disease status beyond first Salvage regimen [HR=1.2 (95\% CI=2.16-3.91); $\mathrm{p}=0.01]$ were independently associated with significant worse survival. Patients with 0 $(n=5), 1(n=26), 2(n=31)$ or $3(n=21)$ adverse factors had a median survival of $42+, 8.8,7.1$, and 2.6 months, respectively (Figure 3 ).

\section{Treatment post inotuzumab ozogamicin}

Thirty-four patients (38\%) received subsequent consolidation therapy with allogeneic stem cell transplantation (ASCT) while five patients received ASCT post inotuzumab failure. Since patients who received ASCT were in good response and well fit, they constituted a selected group with better survival. Their median survival was 9.6 months compared to 3.5 months for those who did not $(\mathrm{p}<0.001)$. Thus, the benefit of inotuzumab was to induce good clinical response that allows patients to benefit from ASCT. Other salvage approaches included: HCVAD regimen $(\mathrm{n}=5)$, fludarabine and cytatarbine-based regimens $(\mathrm{n}=10)$, asparaginase-based regimens $(n=7)$, and other investigational approaches $(n=11)$. 


\section{DISCUSSION}

Inotuzumab ozogamicin has provided a promising new therapeutic option for patients with relapsed and refractory ALL. ${ }^{4-5}$ Our study shows that a marrow CR with or without peripheral blood cell recovery can be achieved in more than $50 \%$ of patients and is associated with survival improvement. Furthermore, responses were observed across the boards in patients with Salvage 1 or Salvage 2 and beyond, although higher rates of responses were observed in patients with less prior therapy. The median survival was 6.3 months, considering the heavily pre-treated population enrolled and is similar to the results reported with blinatumomab in similar patients' population. ${ }^{11}$

In the current analysis we described 2 prognostic scores that can predict survival in patients with relapsed and refractory ALL, at different time points (one at baseline and one after achievement of at least a marrow CR).

The first prognostic score is based on three baseline factors (adverse baseline cytogenetics, disease beyond Salvage 1, high peripheral blood absolute blasts) that were identified through univariate and multivariate analyses. In a series of 609 patients with relapse ALL treated within the MRC UKALL 12/ECOG 2993 study, Fielding and colleagues identified older age and short duration of first remission as adverse fatcors. ${ }^{12}$ Furthermore, and as predicted in the frontline setting, cytogenetic abnormalities play a key role defining prognosis. ${ }^{13-14}$ In our analysis, patients with complex karyotype, $\mathrm{t}(4 ; 11), \mathrm{t}(9 ; 22)$, and abnormal chromosome 17 had worse outcome.

The second prognostic score includes the achievement of at least a marrow CR in addition to 2 baseline characteristics including baseline cytogenetics and disease beyond Salvage 1 . The latter score can be used after re-induction therapy once response status is known. Achieving a response remains a determinant factor for survival and may compensate for other adverse features, as shown in our model. This is similar to our previous report in patients receiving first salvage, where the achievement of CR was associated with better survival outcome. ${ }^{9}$ Conformingly, response to salvage therapy was reported to significant predict for a better outcome in a series of 547 patients with relapse ALL experiencing their first relapse. ${ }^{10}$

The model provided in this analysis is a simple scoring system that identifies a subgroup of patients with relapsed refractory ALL with different prognoses. In patients identified as having poor outcome with single-agent inotuzumab, combinations strategies may be beneficial. Approaches that have demonstrated promise include combination of ALL chemotherapy with monoclonal antibodies, combination of novel tyrosine kinase inhibitors and monoclonal antibodies. Among 30 patients heavily pretreated, the combination of less intensive chemotherapy with inotuzumab ozogamicin has resulted in an objective response rate of 70 and a median survival of 11 months. ${ }^{15}$ Ponatinib is a pan BCR-ABL inhibitor with very potent activity in chronic myeloid leukemia and Philadelphia-positive ALL. The combination of HCVAD with ponatinib has induced durable responses among 37 patients with de novo Ph+ALL with no relapse after a median of 16 months of follow-up. ${ }^{16} \mathrm{~A}$ combination of such TKI with monoclonal antibodies may improve outcome of patients with relapse refractory ALL harboring the $\mathrm{t}(9 ; 22)$. 
In summary, our current analyses identified subsets of adult patients with ALL with different outcomes with inotuzumab ozogamicin. Our results also confirmed the importance of achieving a response to attain improved survival.

\section{References}

1. Mathisen MS, Kantarjian H, Thomas D, O'Brien S, Jabbour E. Acute lymphoblastic leukemia in adults: encouraging developments on the way to higher cure rates. Leuk Lymphoma. 2013; 54(12): 2592-2600. [PubMed: 23547835]

2. Piccaluga PP, Arpinati M, Candoni A, et al. Surface antigens analysis reveals significant expression of candidate targets for immunotherapy in adult acute lymphoid leukemia. Leuk Lymphoma. 2011; 52(2):325-327. [PubMed: 21077738]

3. Thorson JS, Sievers EL, Ahlert J, et al. Understanding and exploiting nature's chemical arsenal: the past, present and future of calicheamicin research. Curr Pharm Des. 2000; 6(18):1841-1879. [PubMed: 11102565]

4. Kantarjian H, Thomas D, Jorgensen J, et al. Inotuzumab ozogamicin, an anti-CD22-calicheamicin conjugate, for refractory and relapsed acute lymphocytic leukaemia: a phase 2 study. Lancet Oncol. 2012; 13:403-411. [PubMed: 22357140]

5. Kantarjian H, Thomas D, Jorgensen J, et al. Results of inotuzumab ozogamicin, a CD22 monoclonal antibody, in refractory and relapsed acute lymphocytic leukemia. Cancer. 2013; 119(15):2728-2736. [PubMed: 23633004]

6. Agresti, A. Categorical Data Analysis. 2. John Wiley \& Sons Inc; Hoboken, NJ: 1990.

7. Kaplan EL, Maier P. Non-parametric estimation from incomplete observations. J Am Stat Assoc. $1965 ; 53: 457-481$.

8. Cox DR. Regression models and life tables. J R Stat Soc. 1972; 34:187-220.

9. Kantarjian HM, Thomas D, Ravandi F, et al. Defining the course and prognosis of adults with acute lymphocytic leukemia in first salvage after induction failure or short first remission duration. Cancer. 2010; 116(24):5568-5574. [PubMed: 20737576]

10. Gökbuget N, Stanze D, Beck J, et al. Outcome of relapsed adult lymphoblastic leukemia depends on response to salvage chemotherapy, prognostic factors, and performance of stem cell transplantation. Blood. 2012; 120(10):2032-2041. [PubMed: 22493293]

11. Topp MS, Goekbuget N, Stein AS, et al. Confirmatory open-label, single-arm, multicenter phase 2 study of the BiTE antibody blinatumomab in patients (pts) with relapsed/refractory B-precursor acute lymphoblastic leukemia (r/r ALL). J Clin Oncol. 2014:325s. abstract 7005.

12. Fielding AK, Richards SM, Chopra R, et al. Outcome of 609 adults after relapse of acute lymphoblastic leukemia (ALL); an MRC UKALL12/ECOG 2993 study. Blood. 2007 Feb 1; 109(3):944-950. [PubMed: 17032921]

13. Pui CH, Evans WE. Treatment of acute lymphoblastic leukemia. N Engl J Med. 2006; 354(2):166178. [PubMed: 16407512]

14. Faderl S, O'Brien S, Pui CH, et al. Adult acute lymphoblastic leukemia: concepts and strategies. Cancer. 2010; 116(5):1165-1176. [PubMed: 20101737]

15. Jabbour E, O'Brien S, Nitin J, et al. Inotuzumab ozogamicin (IO) in combination with lowintensity chemotherapy as front-line therapy for older patients and as salvage therapy for adult with relapse/refractory acute lymphoblastic leukemia. J Clin Oncol. 2014 Abstract \# 7019.

16. O'Brien S, Jabbour E, Thomas D, et al. Phase II study of combination of hyperCVAD with ponatinib in frontline therapy of patients with Philadelphia chromosome positive acute lymphoblastic leukemia. J Clin Oncol. 2014 Abstract \# 7064. 


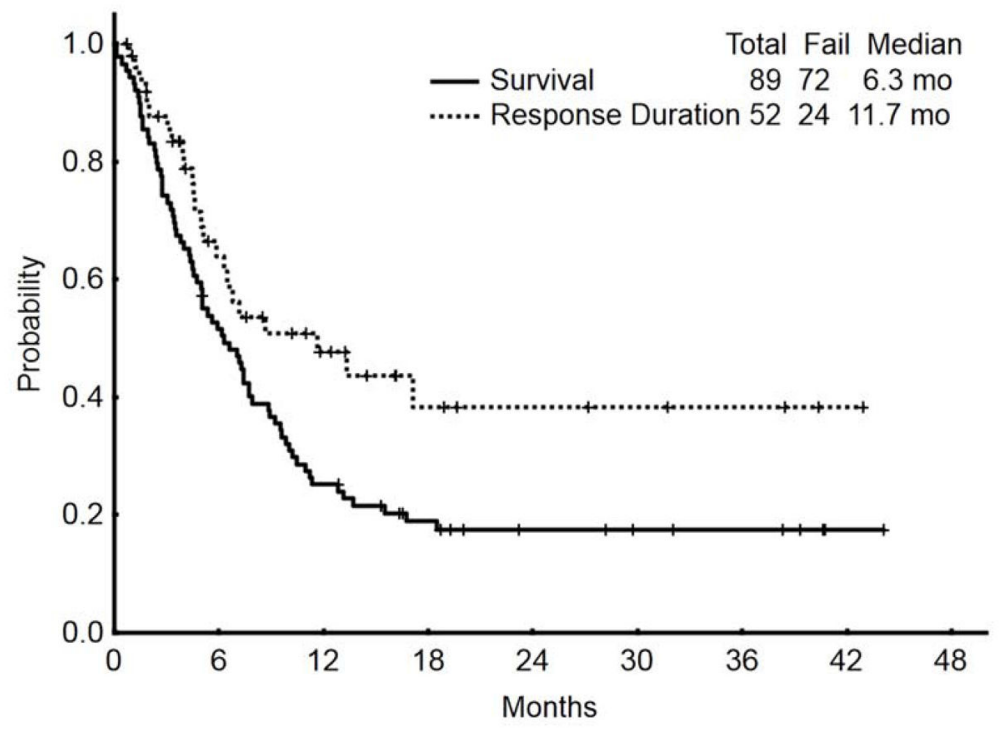

Figure 1.

Survival and response duration for the whole population $(\mathrm{N}=89)$ 


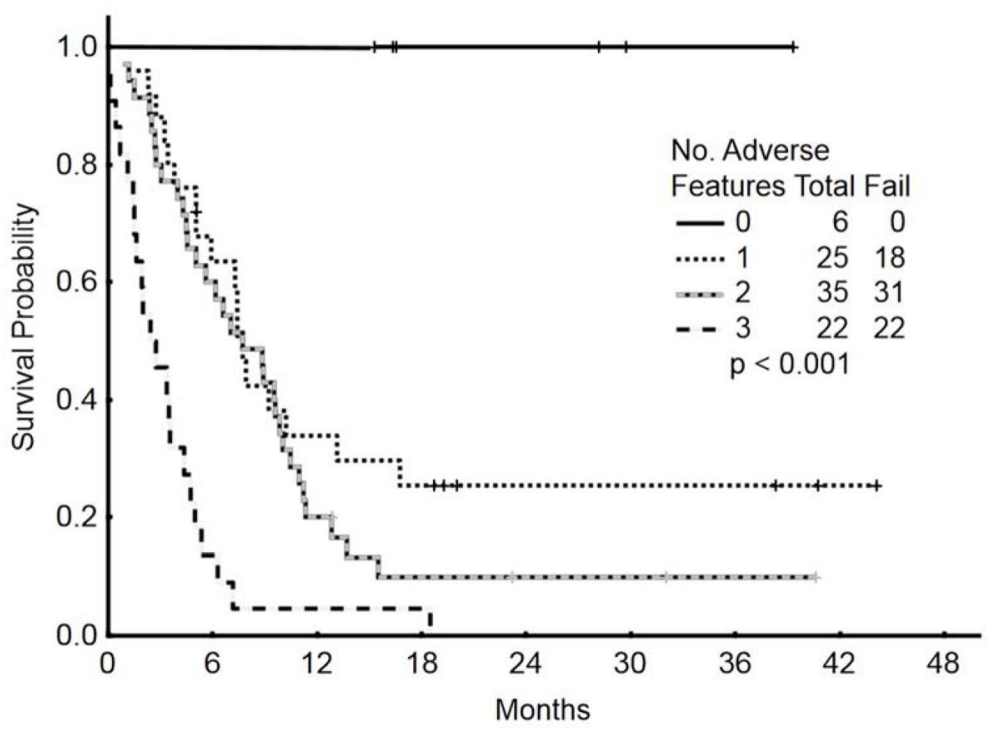

Figure 2.

Survival by risk score according to baseline adverse factors $(\mathrm{N}=88)^{*}$

*One patient had insufficient metaphases and was excluded 


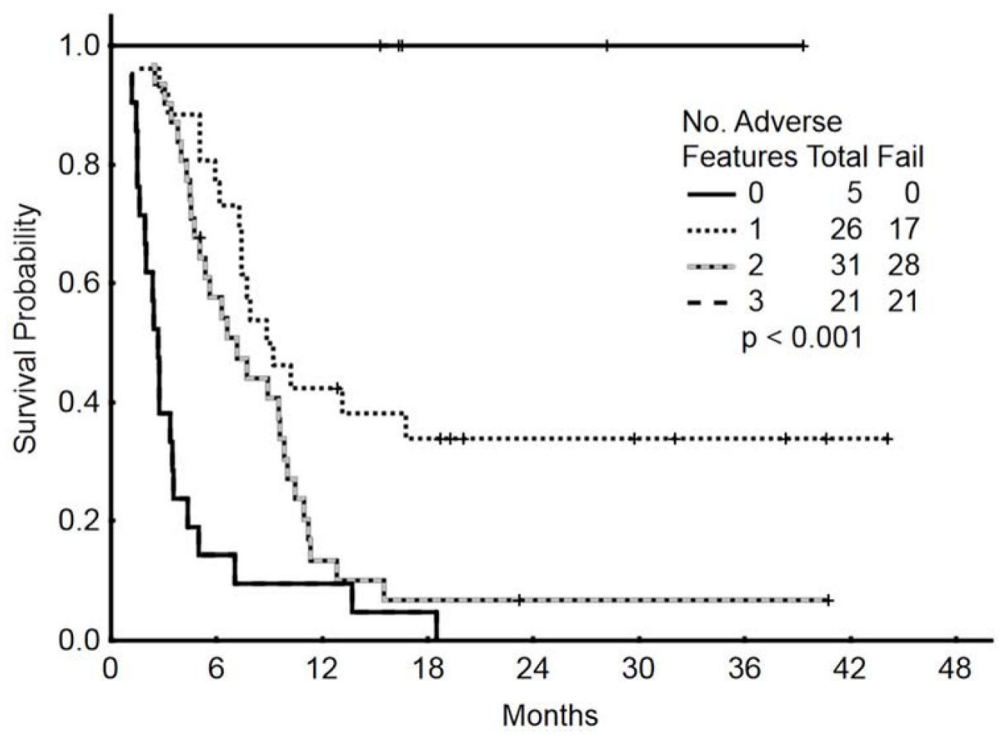

Figure 3.

Survival by risk score according to a landmark analysis taking into account response as an additional variable $(\mathrm{N}=83)$ 
Table 1

Patients' characteristics

\begin{tabular}{|c|c|c|c|c|}
\hline & & \multicolumn{3}{|c|}{$\mathbf{N}(\%)$} \\
\hline Parameter & & Single-Dose, $n=49$ & Weekly, $n=40$ & Overall, $\mathrm{n}=\mathbf{8 9}$ \\
\hline \multirow[t]{2}{*}{ Age, year } & $\leq 8$ & $3(6)$ & $3(8)$ & $6(7)$ \\
\hline & 260 & $12(24)$ & $13(33)$ & $25(28)$ \\
\hline \multirow[t]{2}{*}{ ECOG, PS } & $0-1$ & $44(90)$ & $36(90)$ & $80(90)$ \\
\hline & 22 & $5(10)$ & $4(10)$ & $9(10)$ \\
\hline \multirow[t]{2}{*}{ Salvage Status } & S1 & $13(27)$ & $16(40)$ & $29(33)$ \\
\hline & $>\mathrm{S} 1$ & $36(73)$ & $24(60)$ & $60(67)$ \\
\hline \multirow[t]{2}{*}{$\mathrm{PB} A B C, \times 10^{9} / \mathrm{L}$} & $<1.0$ & $33(67)$ & $25(63)$ & $58(65)$ \\
\hline & $\geq 1.0$ & $16(33)$ & $15(38)$ & $31(35)$ \\
\hline \multirow[t]{3}{*}{ WBC, $\times 10^{9} / \mathrm{L}$} & $<4.0$ & $28(57)$ & $17(43)$ & $45(51)$ \\
\hline & $4-11$ & $11(22)$ & $16(40)$ & $27(30)$ \\
\hline & $>11$ & $10(20)$ & $7(18)$ & $17(19)$ \\
\hline \multirow[t]{4}{*}{ BM blasts, $\%$} & $<20$ & $3(6)$ & $8(20)$ & $11(12)$ \\
\hline & $20-49$ & $10(20)$ & $8(20)$ & $18(20)$ \\
\hline & $50-69$ & $8(16)$ & $6(15)$ & $14(16)$ \\
\hline & $\geq 70$ & $28(57)$ & $18(45)$ & $46(52)$ \\
\hline \multirow[t]{2}{*}{ Platelets, x $10^{9} / \mathrm{L}$} & $<100$ & $43(88)$ & $28(70)$ & $71(80)$ \\
\hline & $\geq 100$ & $6(12)$ & $12(30)$ & $18(20)$ \\
\hline \multirow[t]{6}{*}{ Karyotype $^{*}$} & Diploid & $8(16)$ & $9(23)$ & $17(19)$ \\
\hline & $\mathrm{Ph}$-positive & $9(18)$ & $8(20)$ & $17(19)$ \\
\hline & Translocation $(4 ; 11)$ & $6(12)$ & $3(8)$ & $9(10)$ \\
\hline & Complex & $13(27)$ & $11(28)$ & $24(27)$ \\
\hline & Abnormal chromosome 17 & $6(12)$ & $5(13)$ & $11(12)$ \\
\hline & Other & $6(12)$ & $4(10)$ & $10(11)$ \\
\hline Prior ASCT & & $7(14)$ & $2(5)$ & $9(10)$ \\
\hline \multicolumn{2}{|c|}{ Prior HCVAD regimen } & $35(71)$ & $32(80)$ & $67(75)$ \\
\hline \multirow[t]{3}{*}{ CD22-positive, \% } & $\geq 90$ & $28(57)$ & $31(78)$ & $59(66)$ \\
\hline & $70-89$ & $14(29)$ & $7(18)$ & $21(24)$ \\
\hline & $50-69$ & $7(14)$ & $2(5)$ & $9(10)$ \\
\hline
\end{tabular}

$\mathrm{S}=$ Salvage; $\mathrm{PB}=$ peripheral blood; $\mathrm{ABC}=$ absolute blast count; $\mathrm{BM}=$ bone marrow; $\mathrm{WBC}=$ white blood cell; $\mathrm{PS}=$ =erformance status; ASCT=allogeneic stem cell transplantation

one patient had insufficient metaphases 
Table 2

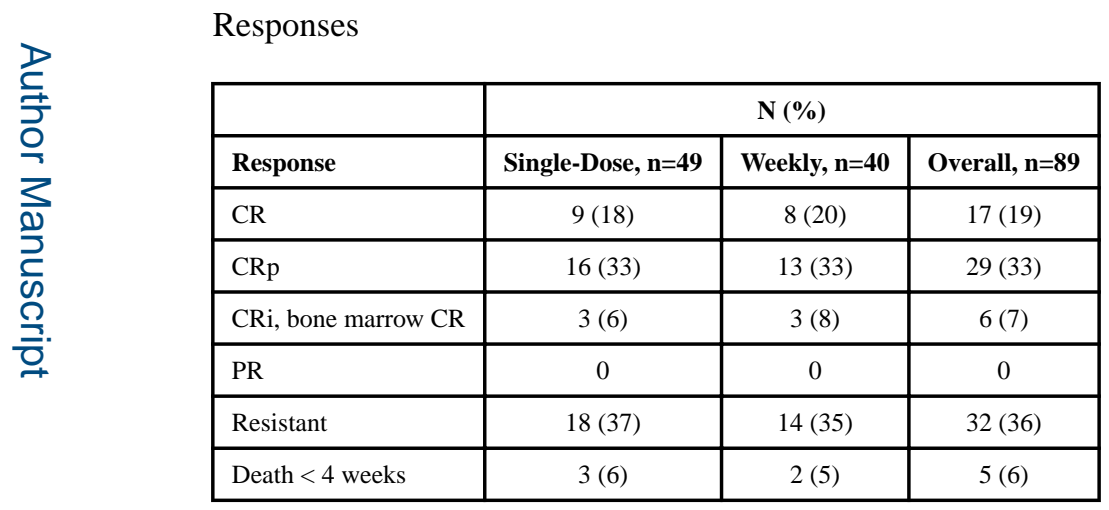

$\mathrm{CR}=$ complete response; $\mathrm{CRp}=$ complete response without platelets recovery; $\mathrm{CRi}=$ bone marrow $\mathrm{CR} ; \mathrm{PR}=$ partial response 


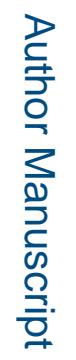

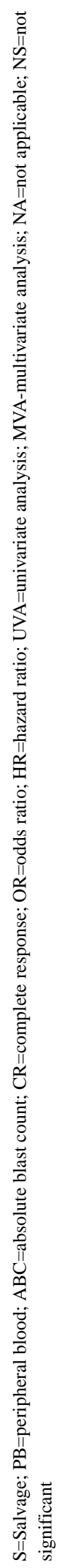

를

\begin{tabular}{|c|c|c|c|c|c|c|}
\hline \multirow{2}{*}{$\sum_{z}^{\mathbb{Z}}$} & $a$ & $\stackrel{5}{\circ}$ & $\tilde{z}$ & $\tilde{o}_{0}^{\circ}$ & $\tilde{z}$ & $\overline{0}_{0}^{0}$ \\
\hline & 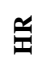 & $\stackrel{m}{i}$ & $\mathbb{z}$ & $\exists$ & $\mathbb{z}$ & $\overrightarrow{\mathrm{i}}$ \\
\hline$\vec{b}$ & a & $\begin{array}{l}\overrightarrow{8} \\
\dot{\theta}\end{array}$ & $\stackrel{\text { ô. }}{0}$ & $\begin{array}{l}\overrightarrow{8} \\
\dot{\theta}\end{array}$ & $\begin{array}{l}\overline{\mathrm{g}} \\
\dot{\vec{v}}\end{array}$ & $\begin{array}{l}\bar{\delta} \\
\dot{0} \\
\dot{v}\end{array}$ \\
\hline
\end{tabular}

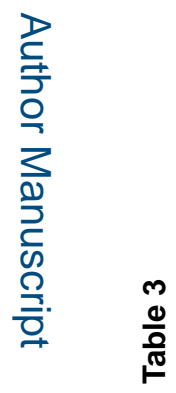

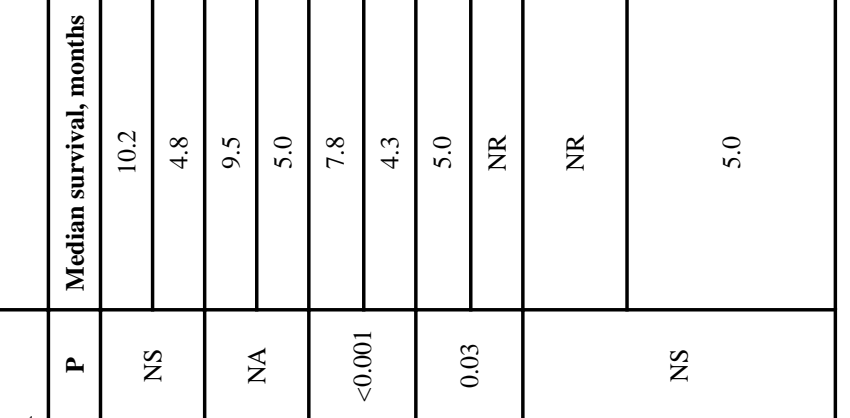

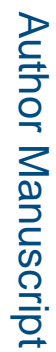

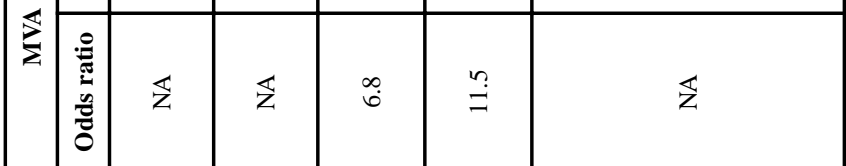

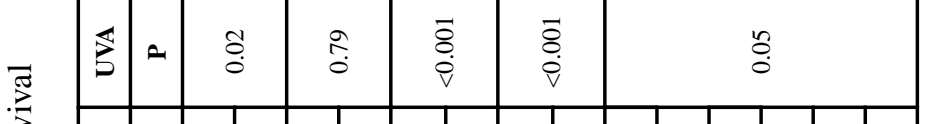

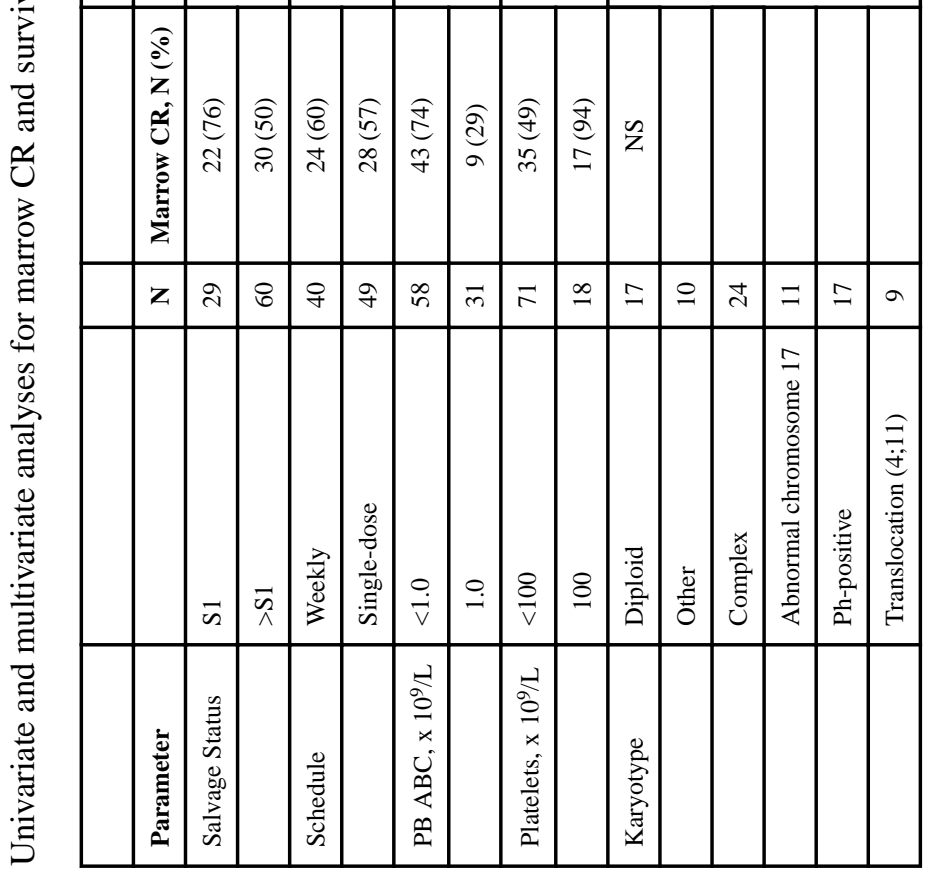

Am J Hematol. Author manuscript; available in PMC 2017 July 11. 